\title{
ON CENTRALIZERS OF $p$-ELEMENTS IN INDECOMPOSABLE MODULES
}

\author{
PETER LANDROCK
}

\begin{abstract}
For $M$ any liftable module of a group algebra of a finite group $\boldsymbol{G}$ over a field of characteristic $p$ and $x \in G$ any $p$-element, we discuss connections between the restriction of $M$ to $\langle x\rangle$ and that of $\chi$ to $\langle x\rangle$, where $\chi$ is the character of $M$. In particular we get a lower bound for the number of Jordan components of this restriction of $M$ in terms of $\chi$ 's restriction. For $M$ a permutation module, this bound is trivially an equality, and we derive several results by $L$. Scott, which hitherto have been considered relatively deep, in a very elementary and straightforward manner.
\end{abstract}

In general if a finite group has a Sylow p-subgroup of nonvanishing order, it is fair to state that to carry through a complete determination of the decomposition numbers in characteristic $p$ is quite demanding and often, with the methods known, even impossible.

One method is to work with Brauer characters rather than modules. The success of this is limited as Brauer characters only determine composition factors. The aim of the present note is to present an idea which in certain situations proves useful and may handle problems where the above method does not work.

If only Brauer characters are in action, only character values on $p$-regular elements are utilized. One point of the following is to obtain information on liftable modules from the character values on $p$-singular elements as well. In particular we obtain some results on permutation characters due to L. Scott [3] by very elementary and straightforeward methods.

In the following we let $(F, R, K)$ be any modular system (notation as in $[3, \mathrm{p}$. 102]) of a finite group $G, F$ of characteristic $p, p$ a divisor of $|G|$. Let $\pi$ denote the maximal ideal of $R$. If $X$ is an $R[G]$-module, we let $\chi_{X}$ denote the character of $X \otimes_{R} K$. Moreover, if $M$ is any liftable $F[G]$-module, we denote by $\hat{M}$ any $R[G]$-module such that $M=\hat{M} / \hat{M} \pi$. By the socle of an $F[G]$-module $M$ we understand the maximal semsimple submodule, and denote it by $\operatorname{soc}(M)$.

$\operatorname{Irr}(G)$ denotes the set of absolutely irreducible characters of $G$. If $B$ is a block of $F[G]$, we denote the corresponding idempotent by $e_{B}$.

If $M$ is an $F[G]$-module and $x \in G$ is a $p$-element, we consider the $F$-linear map $1-x: M \rightarrow M$ defined by $m \rightarrow m(1-x)$. Set

$$
S_{i}(M, x)=\operatorname{ker}(1-x)^{i}, \quad L_{i}(M, x)=\operatorname{Im}(1-x)^{i} .
$$

Received by the editors April 1, 1980.

1980 Mathematics Subject Classification. Primary 20C20; Secondary 20B05. 
Thus $\left\{S_{i}(M, x)\right\}$ form the socle series of $\left.M\right|_{\langle x\rangle}$, and $\left\{L_{i}(M, x)\right\}$ the Loewy series (observe that $L_{0}(M, x)=M$ ). If $P$ is a $p$-group, we set

$$
S_{1}(M, P)=\bigcap_{x \in P} \operatorname{ker}(1-x), \quad L_{1}(M, P)=\sum_{x \in P} \operatorname{Im}(1-x),
$$

which of course equals the socle resp. the radical of $\left.M\right|_{P}$.

In the following, we call $S_{1}(M, x)$ the centralizer of $x$ in $M, S_{1}(M, P)$ the centralizer of $P$ in $M$ and denote their dimensions by $c_{M}(x)$ resp. $c_{M}(P)$.

Let $p^{n}$ equal the order of $x$ and let $r_{i}=r_{i}(M, x)$ denote the number of indecomposable Jordan components of $\left.M\right|_{\langle x\rangle}$ of dimension $i$. Thus

$$
r_{i}=\operatorname{dim}_{F}\left(S_{1}(M, x) \cap L_{i-1}(M, x)\right)-\operatorname{dim}_{F}\left(S_{1}(M, x) \cap L_{i}(M, x)\right)
$$

and

$$
c_{M}(x)=\sum_{i=1}^{p^{n}} r_{i}
$$

where

$$
p^{n} r_{p^{n}}=\operatorname{dim} M-\left(\sum_{i=1}^{p^{n}-1} i r_{i}\right) .
$$

Set $\delta_{M}(x)=\sum_{i=1}^{p^{n}-1} r_{i}$. Then $\delta_{M}(x)=0$ if and only if $\left.M\right|_{\langle x\rangle}$ is projective. From (2) and (3) we get

$$
c_{M}(x)=\delta_{M}(x)+r_{p^{n}}=\frac{1}{p^{n}}\left(\operatorname{dim} M+\sum_{i=1}^{p^{n}-1}\left(p^{n}-i\right) r_{i}\right)
$$

Lemma 1. Let $M$ be any liftable $F[G]-$ module, and let $P<G$ be any $p$-subgroup. Then, for any $\varphi \in \operatorname{Irr}(P)$,

$$
\varphi(1) c_{M}(P)>\left(\chi_{\hat{M}}, \varphi\right)_{P}
$$

Proof. Let $I_{M}$ be the injective hull of $\left.M\right|_{P}$ and let $\chi$ be the character of $\hat{I}_{M}$. Now $\operatorname{soc}\left(I_{M}\right) \simeq \operatorname{soc}\left(\left.M\right|_{P}\right)$ and $I_{M} \simeq F[P]^{n}$ as $F[P]$-modules, where

$$
n=\operatorname{dim}_{F}\left(\operatorname{soc}\left(I_{M}\right)\right)=(\chi, \varphi)_{P} / \varphi(1)
$$

for any $\varphi$ as stated, since $\chi_{R[P]}$ is the regular character of $P$. Thus the statement follows as $\chi=\chi_{\hat{M}}+\chi^{\prime}$ for some character $\chi^{\prime}$.

Unfortunately, the structure of $\left.M\right|_{\langle x\rangle}, x$ any $p$-element, is not determined by $c_{M}(x)$ and $\operatorname{dim}_{F}(M)$ except in one case, namely when $p=2$ and $x$ is an involution, due simply to the fact that any indecomposable $F\left[\mathbf{Z}_{2}\right]$-module is either trivial or free.

However, if we have additional information on $M$ we may still be able to determine $\left.M\right|_{\langle x\rangle}$ from $\chi_{\hat{M}}$. This is the case if $M$ is a direct summand of a permutation module as we shall discuss. We remark that Lemma 2 is independent of Lemma 1. 
LEMMA 2. Let $M$ be a direct summand of a permutation module of $F[G]$, and let $\hat{M}$ be the corresponding $R[G]-$ module (see L. Scott $[3$, Proposition $1(b)]$ ). Let $P=\langle x\rangle$. Then

(i) $c_{M}(x)=\left(\chi_{\hat{M}}, 1_{P}\right)_{P}$

(ii) $\chi_{\hat{M}}(x)$ equals the number of indecomposable components of $\left.\hat{M}\right|_{P}$ isomorphic to the trivial 1-dimensional $F[P]$-module. In particular, $\chi_{\hat{M}}(x)$ is a nonnegative rational integer.

Proof. Let $G$ act on a set $\Omega$ and let $\Delta$ be the corresponding permutation module with $M$ isomorphic to a direct summand. The indecomposable direct summands of $\left.\Delta\right|_{P}$ and in particular of $\left.M\right|_{P}$ are all of the form $N=\operatorname{span}_{F}(S)$, where $S$ is an orbit of $x$ in $\Omega$. Indeed, if $S=\left\{s_{1}, \ldots, s_{n}\right\}$, then $N$ is a uniserial $F[P]$-module with its socle spanned by $\sum_{i=1}^{n} s_{i}$. Hence $c_{N}(x)=1, N$ is a permutation module of $P$, i.e. liftable to $\hat{N}$ with $\left(\chi_{\hat{N}}, 1_{P}\right)=1$, and (i) and (ii) both follow.

Remark. Part (ii) of this lemma is identical with part of Theorem 5 of L. Scott [3]. In fact we may easily obtain all of Theorem 5 of [3] using this result.

COROLlary 1. Same notation as above. Then

(i) $x$ belongs to the vertex of an indecomposable component of $\left.M\right|_{P}$ if and only if $\chi_{\hat{M}}(x)>0$.

(ii) Let $B$ be a block of $F[G]$ with defect group $P$, and let $M$ be any indecomposable component of $\Delta e_{B}$. Then $x^{i}$ belongs to the vertex of $M$ if and only if $\chi_{\hat{M}}\left(x^{i}\right)>0$.

Proof. (i) follows from (ii) of Lemma 2, while (ii) follows from (i) since the vertex of $M$ in this case a priori is a subgroup of $\langle x\rangle$.

Corollary 2 (L. Scott, [3, Corollary B of Theorem 7]). Let $B$ be a block of $F[G]$ with defect group $\langle x\rangle \simeq \mathbf{Z}_{p}, F$ a splitting field, and let $b$ be the corresponding block of $N:=N_{G}(\langle x\rangle)$. Furthermore we denote by $\left\{M_{i}\right\}$ the set of indecomposable components of $\Delta e_{B}$ which are nonprojective, $\left\{f\left(M_{i}\right)\right\}$ their Green correspondents in $N$. Let $\left\{\hat{M}_{i}\right\}$ and $\left\{f\left(\hat{M}_{i}\right)\right\}$ be the corresponding modules over $R$. Then

(i) $f\left(M_{i}\right)$ has $\langle x\rangle$ in its kernel.

(ii) $\chi_{f\left(\hat{M}_{i}\right)}$ is an irreducible character of $N$ and $\chi_{\hat{M}}(x)=\chi_{f(\hat{M})}(1)$.

(iii) Let $\Gamma$ be the $F[N]$-module spanned by the fixpoints of $x$ in $\Omega$. Then

$$
\Gamma e_{b}=\sum_{i} f\left(M_{i}\right)
$$

(iv) $\chi_{\hat{M}}$ is either irreducible nonexceptional or the sum of all exceptional characters of $B$.

Proof. The fact that $\langle x\rangle \simeq \mathbf{Z}_{p}$ implies that any indecomposable component of $\left.\Delta\right|_{\langle x\rangle}$ is either trivial or projective. Hence $M_{i}$ and $f\left(M_{i}\right)$ have the trivial 1-dimensional $\langle x\rangle$-module as source, and (i) follows. Thus $f\left(M_{i}\right)$ is an indecomposable $F[N /\langle x\rangle]$-module in a block of defect 0 , i.e., projective with $\chi_{f\left(\hat{M}_{i}\right)}$ irreducible, proving (ii).

Now, let $\left.\Delta\right|_{\langle x\rangle}=\Delta_{1} \oplus \Delta_{2}$, where all components of $\Delta_{1}$ are trivial, corresponding to fixpoints of $x$, and all components of $\Delta_{2}$ are projective corresponding to orbits of 
$x$ of length $p$. Then $\Delta_{1}$ in fact is an $F[N]$-module, and $\Delta_{1} \simeq \Gamma$ as $F[N]$-modules, from which (iii) follows.

(iv) immediately follows from (ii) and (iii), using the theory of blocks with cyclic defect groups. See W. Feit [1, Chapter VI, (5.8)].

We next turn to arbitrary liftable modules.

TheOREM 1. Let $M$ be any liftable module, and let $\chi$ be the character of $M$. Assume that $x \in G$ is a p-element with $\left.\chi\right|_{\langle x\rangle}$ rational. Set $P_{i}=\left\langle x^{p^{\prime}}\right\rangle$. Then

$$
c_{M}(x)>\max _{i}\left\{\frac{1}{\left|P: P_{i}\right|}\left(\left(x, 1_{P_{i}}\right)_{P_{i}}+\delta\left|x\left(x^{p^{p-1}}\right)\right|\right)\right\}
$$

where $\delta=1$ for $i \neq 0, \delta=0$ for $i=0$.

Proof. As $\left.\chi\right|_{\langle x\rangle}$ is rational by assumption, $\chi$ is constant on $P_{i} / P_{i+1}$ for all $i$. Let $1_{P} \neq \varphi$ be an irreducible character of $\langle x\rangle$ with kernel $P_{i}$. Then

$$
\sum_{y \in P_{i-1} \backslash P_{i}} \varphi(y)=-\left|P_{i}\right|
$$

while

$$
\sum_{y \in P_{j-1} \backslash P_{j}} \varphi(y)=0
$$

for $j<i$. Thus if $P_{i}<P$,

$$
(\chi, \varphi)_{P}=\frac{1}{|P|}\left(\sum_{y \in P_{i}} \chi(y)-\left|P_{i}\right| \chi\left(x^{p^{i-1}}\right)\right) .
$$

If $\chi\left(x^{p^{i-1}}\right)<0$, this equals

$$
\left(1 /\left|P: P_{i}\right|\right)\left(\left(\chi, 1_{P_{i}}\right)_{P_{t}}+\left|\chi\left(x^{p^{i-1}}\right)\right|\right) \text {. }
$$

Assume therefore that $\chi\left(x^{p^{i-1}}\right)>0$. Choose $j<i-1$ minimal, so that $\chi\left(x^{j}\right)>0$. Then

$$
\begin{aligned}
\frac{1}{|P|}\left(\sum_{y \in P_{i}} \chi(y)+\left|P_{i}\right| \chi\left(x^{p^{i-1}}\right)\right) & <\frac{1}{|P|}\left(\sum_{y \in P_{i}} \chi(y)+\left|P_{i}\right|(p-1) \chi\left(x^{p^{i-1}}\right)\right) \\
& =\frac{1}{|P|} \sum_{y \in P_{i-1}} \chi(y)<\frac{1}{|P|} \sum_{y \in P_{j}} \chi(y) .
\end{aligned}
$$

If $j=0$, this equals $\left(\chi, 1_{P}\right)_{P}$. If $j>0$, we obtain from (10)

$$
\frac{1}{|P|}\left(\sum_{y \in P_{i}} x(y)+\left|P_{i}\right| \chi\left(x^{p^{i-1}}\right)\right)<\frac{1}{|P|}\left(\sum_{y \in P_{j}} \chi(y)-\left|P_{j}\right| \chi\left(x^{p^{j-1}}\right)\right)
$$

since $\chi\left(x^{p^{j-1}}\right)<0$ by assumption. Thus

$$
\max _{\varphi}(\chi, \varphi)_{P}=\max _{i}\left\{\frac{1}{\left|P: P_{i}\right|}\left(\left(\chi, 1_{P_{i}}\right)_{P_{i}}+\delta\left|\chi\left(x^{P^{i-1}}\right)\right|\right)\right\}
$$

and the theorem follows from Lemma 1. 
Corollary 3. Let as before $r_{i}$ denote the number of indecompasable Jordan components of dimension $i$ of $\left.M\right|_{\langle x\rangle}$. Then

$$
\sum_{i=1}^{p^{n-1}}\left(p^{n}-i\right) r_{i} \geqslant \max _{i}\left(\sum_{y \in P_{i}} \chi(y)+\delta\left|P_{i}\right|\left|\chi\left(x^{p^{i-1}}\right)\right|\right) .
$$

Proof. By (4).

COROLLARY 4. Let $p=2$, and let $\chi$ be any character of $G, \hat{M}$ an $R$-form of $\chi$ and $M=\hat{M} / \hat{M} \pi$. Let $\tau$ be any involution in $G$. Then

$$
c_{M}(\tau)>(\chi(1)+|\chi(\tau)|) / 2
$$

Proof. Most of the computations above are superfluous to obtain this very elementary result.

REMARK. This result has been applied in P. Landrock and G. O. Michler [2] to determine the exact structure of $\boldsymbol{R}$-forms of irreducible characters restricted to the centralizer of an involution. The reader is referred to this paper for examples. The main application is the following: If $\tau$ is central, all indecomposable modules are $C_{G}(\tau)$-projective, and finding their restrictions to this group will usually enable one to determine their Green correspondents. Now if $G$ has a large subgroup containing $\tau$ it may be possible to determine $c_{M}(\tau)$ inside this group, and (15) may be applied to all block components of $\left.M\right|_{C_{G}(\tau)}$.

It seems to be an empirical fact that in most cases one may find an $R$-form such that equality holds in (15). This, however, is not a general fact, as was pointed out to the author by W. Plesken. The natural 4-dimensional representation of $\mathbf{Z}_{2} \sim \Sigma_{4}$ provides a counterexample for an involution in the normal elementary abelian subgroup of order 16 with trace 0.

Finally we point out that even though Theorem 1 only describes a liftable module, it may be combined with (4) to obtain information on submodules and factor modules as well. Indeed, if

$$
0 \rightarrow M^{\prime} \rightarrow M \rightarrow M^{\prime \prime} \rightarrow 0
$$

is any short exact sequence, we obviously have, with the notation preceding Lemma 1,

$$
r_{0}=r_{p^{n}}(M, x)-r_{p^{n}}\left(M^{\prime}, x\right)-r_{p^{n}}\left(M^{\prime \prime}, x\right)>0
$$

and

$$
\max \left\{\delta_{M^{\prime}}(x), \delta_{M^{\prime \prime}}(x)\right\} \leqslant \delta_{M^{\prime}}(x)+r_{0}<\delta_{M^{\prime}}(x)+\delta_{M^{\prime \prime}}(x)
$$

thus relating $\delta_{M}(x)$ with $\delta_{M^{\prime}}(x)$ and $\delta_{M^{\prime \prime}}(x)$.

\section{REFERENCES}

1. W. Feit, Representations of finite groups, Lecture Notes, Yale University, New Haven, Conn., 1969-1975.

2. P. Landrock and G. O. Michler, Principal 2-blocks of the simple groups of Ree type, Trans. Amer. Math. Soc. 260 (1980), 83-111.

3. L. L. Scott, Modular permutation representations, Trans. Amer. Math. Soc. 175 (1973), 101-122.

Matematisk Institut, Aarhus Universitet, 8000 AARhus C, Denmark 